\title{
A Note on $k$-Potence Preservers on Matrix Spaces over Complex Field
}

\author{
Xiaofei Song, ${ }^{1}$ Chongguang Cao, ${ }^{2}$ and Baodong Zheng ${ }^{1}$ \\ ${ }^{1}$ Department of Mathematics, Harbin Institute of Technology, Harbin 150001, China \\ ${ }^{2}$ School of Mathematical Sciences, Heilongjiang University, Harbin 150080, China \\ Correspondence should be addressed to Baodong Zheng; zbd@hit.edu.cn
}

Received 13 March 2013; Accepted 3 May 2013

Academic Editor: Chunrui Zhang

Copyright (C) 2013 Xiaofei Song et al. This is an open access article distributed under the Creative Commons Attribution License, which permits unrestricted use, distribution, and reproduction in any medium, provided the original work is properly cited.

Let $\mathbb{C}$ be the field of all complex numbers, $M_{n}$ the space of all $n \times n$ matrices over $\mathbb{C}$, and $S_{n}$ the subspace of $M_{n}$ consisting of all symmetric matrices. The map $\phi: S_{n} \rightarrow M_{n}$ satisfies that $A-\lambda B$ is $k$-potent in $S_{n}$ implying that $\phi(A)-\lambda \phi(B)$ is $k$-potent in $M_{n}$, where $\lambda \in \mathbb{C}$, then there exist an invertible matrix $P \in M_{n}$ and $\epsilon \in \mathbb{C}$ with $\epsilon^{k}=\epsilon$ such that $\phi(X)=\epsilon P^{-1}(X) P$ for every $X \in S_{n}$. Moreover, the inductive method used in this paper can be used to characterise similar maps from $M_{n}$ to $M_{n}$.

\section{Introduction}

Let $\mathbb{C}$ be the field of all complex numbers, $M_{n}$ the space of all $n \times n$ matrices over $\mathbb{C}, T_{n}$ the subspace of $M_{n}$ consisting of all triangular matrices, and $S_{n}$ the subspace of $M_{n}$ consisting of all symmetric matrices. For fixed integer $k \geq 2, A \in$ $M_{n}$ is called a $k$-potent matrix if $A^{k}=A$; especially, $A$ is an idempotent matrix when $k=2$. The map $\phi$ : $S_{n} \rightarrow M_{n}$ satisfies that $A-\lambda B$ is a $k$-potent matrix in $S_{n}$ implying that $\phi(A)-\lambda \phi(B)$ is a $k$-potent matrix in $M_{n}$, where $\lambda \in \mathbb{C}$, is a kind of the so-called weak preservers. While replacing "implying that" with "if and only if," $\phi$ is called strong preserver. Obviously, a strong preserver must be a weak preserver, while a weak preserver may not be a strong preserver.

The preserver problem in this paper is from LPPs but without linear assumption (more details about LPP in [1-3]). You and Wang characterized the strong $k$-potence preservers from $M_{n}$ to $M_{n}$ in [4]; then Song and Cao extended the result to weak preservers from $M_{n}$ to $M_{n}$ in [5]. In [6], Wang and You characterized the strong $k$-potence preservers from $T_{n}$ to $M_{n}$. In this paper, the authors characterized the weak $k$ potence preservers from $S_{n}$ to $M_{n}$ and proved the following theorem.
Theorem 1. Suppose $\phi: S_{n} \rightarrow M_{n}$ satisfy that $A-\lambda B$ is a $k$-potent matrix in $S_{n}$ implying that $\phi(A)-\lambda \phi(B)$ is a $k$-potent matrix in $M_{n}$, where $\lambda \in \mathbb{C}$. Then there exist invertible $P \in M_{n}$ and $\epsilon \in \mathbb{C}$ with $\epsilon^{k}=\epsilon$ such that $\phi(X)=\epsilon P^{-1} X P$ for every $X \in S_{n}$.

Furthermore, we can derive the following corollary from Theorem 1.

Corollary 2. Suppose $\phi: S_{n} \rightarrow S_{n}$ satisfy that $A-\lambda B$ is a $k$-potent matrix in $S_{n}$ implying that $\phi(A)-\lambda \phi(B)$ is a $k$-potent matrix in $S_{n}$, where $\lambda \in \mathbb{C}$. Then there exist invertible $P \in M_{n}$ and $\epsilon \in \mathbb{C}$ with $\epsilon^{k}=\epsilon$ such that $\phi(X)=\epsilon P^{-1} X P$ for every $X \in S_{n}$, where $P P^{t}=a I_{n}$ for some nonzero $a \in \mathbb{C}$.

In fact, the proof of Theorem 1 through some adjustments is suitable for the weak $k$-potence preserver from $M_{n}$ to $M_{n}$, and more details can be seen in remarks.

\section{Notations and Lemmas}

$\Gamma_{n}$ denotes the set of all $k$-potent matrices in $M_{n}$, while $S \Gamma_{n}=$ $\Gamma_{n} \cap S_{n} . \Lambda$ denotes the set of all complex number $\epsilon$ satisfying $\epsilon^{k-1}=1, \Delta=\Lambda \cup\{0\} . E_{i j}$ denotes matrices in $M_{n}$ with 1 in 
$(i, j)$ and 0 elsewhere, and $I_{n}$ denotes the unit matrix in $M_{n}$. $\langle n\rangle$ denotes the set of integer $s$ satisfy $1 \leq s \leq n$. GL $L_{n}$ denotes the general linear group consisting of all invertible matrices in $M_{n}$. $D_{n}$ denotes an arbitrary diagonal matrix in $M_{n}$. For $A, B \in M_{n}, A$ and $B$ are orthogonal if $A B=B A=0 . \mathbb{C}^{n \times 1}$ denotes the space of all $n \times 1$ matrices over $\mathbb{C}$. $\Phi_{n}$ denotes the set of all maps $\phi: S_{n} \rightarrow M_{n}$ satisfying that $A-\lambda B$ is a $k$ potent matrix in $S_{n}$ implying that $\phi(A)-\lambda \phi(B)$ is a $k$-potent matrix in $M_{n}$, where $\lambda \in \mathbb{C}$.

For an arbitrary matrix $X \in M_{n}$, we denote by $X[i, j]$ the term in $(i, j)$ position of $X$, by $X_{\left[i_{1}, \ldots, i_{s} ; j_{1}, \ldots, j_{t}\right]}$ the $s \times t$ matrix with the term in its $(p, q)$ position equal to $X\left[i_{p}, j_{q}\right]$, where $i_{1}<\cdots<i_{s}$ and $j_{1}<\cdots<j_{t}$. Moreover, we denote by $X_{\left\{i_{1}, \ldots, i_{s} ; j_{1}, \ldots, j_{t}\right\}}$ the $n \times n$ matrix with the term in its $\left(i_{p}, j_{q}\right)$ position equal to $X\left[i_{p}, j_{q}\right]$ and terms elsewhere equal to 0 . We especially simplify it with $X_{\left\{i_{1}, \ldots, i_{s}\right\}}$ when $s=t$, and $i_{l}=j_{l}$ for every $l \in\langle s\rangle$. Naturally, $X_{\{i\}}=X[i, i] E_{i i}$ for every $i \in\langle n\rangle$.

Without fixing $X, X_{\left\{i_{1}, \ldots, i_{s} ; j_{1}, \ldots, j_{t}\right\}}$ also denotes a matrix in $M_{n}$ with 0 in its $(p, q)$ position, where $p \notin\left\{i_{1}, \ldots, i_{s}\right\}, q \notin$ $\left\{j_{1}, \ldots, j_{t}\right\}$, and $1 \leq i_{1}<\cdots<i_{s} \leq n, 1 \leq j_{1}<\cdots<j_{t} \leq n$.

At first, we need the following Lemmas 3, 4, 5, and 7, which are about $k$-potent matrices and orthogonal matrices.

Lemma 3 (see [2]). Suppose $X, Y \in \Gamma_{n}$, and $X+\epsilon Y \in \Gamma_{n}$ for every $\epsilon \in \Lambda$; then $X$ and $Y$ are orthogonal.

Lemma 4 ([7, Lemma 1]). Suppose $A_{1}, A_{2}, \ldots, A_{n}$ are $n \times$ $n$ mutually orthogonal nonzero $k$-potent matrices; then there exists $P \in G L_{n}$ such that $P^{-1} A_{i} P=c_{i} E_{i i}$ with $c_{i}^{k-1}=1$ for every $i \in\langle n\rangle$.

Lemma 5. Suppose $Z \in M_{n-1}, p, q, g, h \in \mathbb{C}^{(n-1) \times 1}$ with $g h^{t} \neq 0, \delta \in \mathbb{C}$, for arbitrary nonzero $\alpha \in \mathbb{C}$ with $h^{t} g+\alpha^{2} \neq 0$ and $\tau=\left(\alpha^{-1} h^{t} g+\alpha\right)^{-1}, \tau\left[\begin{array}{ll}Z & p \\ q^{t} \delta\end{array}\right]+\tau\left[\begin{array}{ll}\alpha^{-1} g h^{t} & g \\ h^{t} & \alpha\end{array}\right] \in \Gamma_{n}$. Then $Z=0, \delta=0$, and there exist $\lambda_{1}, \lambda_{2} \in \mathbb{C}$ with $\left(\lambda_{1}+1\right)\left(\lambda_{2}+1\right)=$ 1 such that $p=\lambda_{1} g$ and $q=\lambda_{2} h$.

Proof. By the assumption of $\alpha$ and $\tau, \tau\left[\begin{array}{ll}\alpha^{-1} g h^{t} & g \\ h^{t} & \alpha\end{array}\right]$ is idempotent. Denote this matrix by $X$, and then we can get the following equation:

$$
\left[\begin{array}{lc}
I_{n-1} & -\alpha^{-1} g \\
\tau h^{t} & 1-\tau \alpha^{-1} h^{t} g
\end{array}\right] X\left[\begin{array}{ll}
I_{n-1}-\tau \alpha^{-1} g h^{t} & \alpha^{-1} g \\
-\tau h^{t} & 1
\end{array}\right]=\left[\begin{array}{ll}
0 & 0 \\
0 & 1
\end{array}\right] .
$$

Since the matrices on both sides of $X$ satisfy the following equation:

$$
\left[\begin{array}{lc}
I_{n-1} & -\alpha^{-1} g \\
\tau h^{t} & 1-\tau \alpha^{-1} h^{t} g
\end{array}\right]\left[\begin{array}{ll}
I_{n-1}-\tau \alpha^{-1} g h^{t} & \alpha^{-1} g \\
-\tau h^{t} & 1
\end{array}\right]=I_{n}
$$

then the following matrix is $k$-potent by the assumption of lemma:

$$
\begin{gathered}
{\left[\begin{array}{cc}
I_{n-1} & -\alpha^{-1} g \\
\tau h^{t} & 1-\tau \alpha^{-1} h^{t} g
\end{array}\right]\left(\tau\left[\begin{array}{ll}
Z & p \\
q^{t} & \delta
\end{array}\right]+X\right)} \\
\times\left[\begin{array}{ll}
I_{n-1}-\tau \alpha^{-1} g h^{t} & \alpha^{-1} g \\
-\tau h^{t} & 1
\end{array}\right] .
\end{gathered}
$$

We denote by $A$ the following matrix:

$$
\left[\begin{array}{lc}
I_{n-1} & -\alpha^{-1} g \\
\tau h^{t} & 1-\tau \alpha^{-1} h^{t} g
\end{array}\right]\left[\begin{array}{ll}
Z & p \\
q^{t} & \delta
\end{array}\right]\left[\begin{array}{ll}
I_{n-1}-\tau \alpha^{-1} g h^{t} & \alpha^{-1} g \\
-\tau h^{t} & 1
\end{array}\right]
$$

then the following equation is obvious:

$$
\left(\tau A+\left[\begin{array}{ll}
0 & 0 \\
0 & 1
\end{array}\right]\right)^{k}=\tau A+\left[\begin{array}{ll}
0 & 0 \\
0 & 1
\end{array}\right] \text {. }
$$

Unfolding it, we get $\tau^{k} A^{k}+\tau^{k-1}(\cdots)_{k-1}+\cdots+\tau(\cdots)_{1}+\left[\begin{array}{ll}0 & 0 \\ 0 & 1\end{array}\right]=$ $\tau A+\left[\begin{array}{ll}0 & 0 \\ 0 & 1\end{array}\right]$; that is, $\tau^{k} A^{k}+\tau^{k-1}(\cdots)_{k-1}+\cdots+\tau(\cdots)_{1}-\tau A=0$, where $(\cdots){ }_{i}$ is the coefficient matrix of $\tau^{i}$ for every $i \in\langle k-1\rangle$.

Let $A=\left[\begin{array}{ll}Z_{1} & p_{1} \\ q_{1}^{t} & \delta_{1}\end{array}\right]$, then we calculate it and get the following equations:

$$
\begin{gathered}
Z_{1}=\left(Z-\alpha^{-1} g q^{t}\right)\left(I_{n-1}-\tau \alpha^{-1} g h^{t}\right)-\left(p-\delta \alpha^{-1} g\right) \tau h^{t} \\
p_{1}=\left(Z-\alpha^{-1} g q^{t}\right) \alpha^{-1} g+p-\delta \alpha^{-1} g \\
q_{1}^{t}=\left(\tau h^{t} Z+q^{t}-\tau \alpha^{-1} q^{t} h^{t} g\right)\left(I_{n-1}-\tau \alpha^{-1} g h^{t}\right) \\
-\left(\tau h^{t} p+\delta-\delta \tau \alpha^{-1} h^{t} g\right) \tau h^{t} \\
\delta_{1}=\left(\tau h^{t} Z+q^{t}-\tau \alpha^{-1} q^{t} h^{t} g\right) \alpha^{-1} g \\
+\tau h^{t} p+\delta-\delta \tau \alpha^{-1} h^{t} g .
\end{gathered}
$$

It is easy to get $\tau(\cdots)_{1}=\tau\left[\begin{array}{cc}0 & p_{1} \\ q_{1}^{t} & k \delta_{1}\end{array}\right]$ and the following equation:

$$
\begin{gathered}
\tau^{k} A^{k}+\tau^{k-1}(\cdots)_{k-1}+\cdots+\tau^{2}(\cdots)_{2} \\
+\tau\left[\begin{array}{cc}
-Z_{1} & 0 \\
0 & (k-1) \delta_{1}
\end{array}\right]=0
\end{gathered}
$$

Note that the highest degree of $\alpha$ in $\tau^{-2} A$ is 2; then the highest degree of $\alpha$ in $\tau^{-3 k+i}(\cdots)_{k-i}$ is less or equal to $3 k-i$ for every $i$ with $2 \leq i \leq k-1$, and the highest degree of $\alpha$ in $\tau^{-3 k+1}\left[\begin{array}{cc}-Z_{1} & 0 \\ 0 & (k-1) \delta_{1}\end{array}\right]$ is $3 k-1$, where $Z$ is the coefficient matrix of $\alpha^{3 k-1}$ in $Z_{1}$ and $\delta$ is the coefficient of $\alpha^{3 k-1}$ in $\delta_{1}$.

By the assumption of $\alpha$, we have $Z=0$ and $\delta=0$. Then the following equations are true:

$$
\begin{gathered}
Z_{1}=-\alpha^{-1} g q^{t}\left(I_{n-1}-\tau \alpha^{-1} g h^{t}\right)-p \tau h^{t}, \\
p_{1}=-\alpha^{-1} g q^{t} \alpha^{-1} g+p, \\
q_{1}^{t}=\left(q^{t}-\tau \alpha^{-1} q^{t} h^{t} g\right)\left(I_{n-1}-\tau \alpha^{-1} g h^{t}\right)-\tau h^{t} p \tau h^{t}, \\
\delta_{1}=\left(q^{t}-\tau \alpha^{-1} q^{t} h^{t} g\right) \alpha^{-1} g+\tau h^{t} p
\end{gathered}
$$

and $\tau^{-3 k+1} Z_{1}=\tau^{-3 k+1}\left[-\alpha^{-1} g q^{t}+\alpha^{-1} g q^{t} \tau \alpha^{-1} g h^{t}-p \tau h^{t}\right]=$ $\tau^{-3 k+2}\left[-\tau^{-1} \alpha^{-1} g q^{t}+\alpha^{-2} g q^{t} g h^{t}-p h^{t}\right]$, where the highest degree of $\alpha$ is $3 k-2$ and $-g q^{t}-p h^{t}$ is the coefficient matrix of $\alpha^{3 k-2}$. 
Now, we calculate the upper left part of $\tau^{-3 k+2}(\cdots)_{2}$.

When $k=2, \tau^{-3 k+2}(\cdots)_{2}=\tau^{-4} A^{2}$, of which the upper left part is $\tau^{-4}\left[p q^{t}\left(I_{n-1}-\tau \alpha^{-1} g h^{t}\right)-q^{t} p \alpha^{-1} g \tau h^{t}\right]=\tau^{-4}\left[p q^{t}-\right.$ $\left.\tau \alpha^{-1} p q^{t} g h^{t}-\tau \alpha^{-1} q^{t} p g h^{t}\right]$. Then in the upper left part of $\tau^{-4} A^{2}+\tau^{-5}\left[\begin{array}{cc}-Z_{1} & 0 \\ 0 & (k-1) \delta_{1}\end{array}\right]$, the highest degree of $\alpha$ is 4 , and the coefficient matrix is $p q^{t}+g q^{t}+p h^{t}$.

When $k>2$, if $\left[\begin{array}{ll}0 & 0 \\ 0 & 1\end{array}\right]$ appears in the left (or right) end of an additive item of $\tau^{-3 k+2}(\cdots)_{2}$, then the upper left part of this item is 0 . So, the upper left part of $\tau^{-3 k+2}(\cdots)_{2}$ is equal to the upper left part of $\tau^{-3 k+2} A\left[\begin{array}{ll}0 & 0 \\ 0 & 1\end{array}\right]^{k-2} A$; that is, the upper left part is $\tau^{-3 k+2} p_{1} q_{1}^{t}=\tau^{-3 k+4}\left[\tau^{-1} \alpha p q^{t}-\left(q^{t} g+h^{t} p\right) p h^{t}-\right.$ $\left.\tau^{-1} \alpha^{-1} g q^{t} g q^{t}+\alpha^{-2} q^{t} g\left(q^{t} g+h^{t} p\right) g h^{t}\right]$, and the highest degree of $\alpha$ is $3 k-2$ with $p q^{t}$ as the coefficient matrix of $\alpha^{3 k-2}$.

By the assumption of $\alpha$, we have $p q^{t}+g q^{t}+p h^{t}=0$.

By $g h^{t} \neq 0$, we have $g \neq 0, h \neq 0$, and $p=0$ if and only if $q=0$. When $p \neq 0$, we can get $p=\lambda_{1} g$ by $p\left(q^{t}+h^{t}\right)+g q^{t}=0$, and $q=\lambda_{2} h$ by $(p+g) q^{t}+p h^{t}=0$, where $\lambda_{1}$ and $\lambda_{2}$ satisfy $\lambda_{1} \lambda_{2} g h^{t}+\lambda_{2} g h^{t}+\lambda_{1} g h^{t}=0$; that is, $\lambda_{1} \lambda_{2}+\lambda_{2}+\lambda_{1}=0$ by $g h^{t} \neq 0$, which is equivalent to $\left(\lambda_{1}+1\right)\left(\lambda_{2}+1\right)=1$. When $p=q=0, \lambda_{1}=\lambda_{2}=0$.

Remark 6. Replacing $g h^{t} \neq 0$ with $g h^{t}=0$ in Lemma 5, we have $g=0$ implies $p=0$ or $q+h=0$, and $h=0$ implies $q=0$ or $p+g=0$. These cases will not appear in the proof of Theorem 1, but are necessary for the weak preservers from $M_{n}$ to $M_{n}$.

Lemma 7. Suppose $A=\left[\begin{array}{cc}0 & (\lambda(a)-\lambda(b)) /(a-b) \\ \left(\lambda(a)^{-1}-\lambda(b)^{-1}\right) /(a-b) & 1\end{array}\right] \epsilon$ $\Gamma_{2}$ for arbitrary $a, b \in \mathbb{C}$ with $a \neq b$, where $\lambda: \mathbb{C} \rightarrow \mathbb{C}$ is a map satisfying $\lambda(x) \neq 0$ for every $x \in \mathbb{C}$. Then there exists nonzero $\lambda_{0} \in \mathbb{C}$ such that $\lambda(x)=\lambda_{0}$ for every $x \in \mathbb{C}$.

Proof. Since the trace of $A$ is equal to 1 , then $(\lambda(a)-$ $\lambda(b))\left(\lambda^{-1}(a)-\lambda^{-1}(b)\right) /(a-b)^{2}=0$, or -1 , especially, when equal to $-1, k-1=6 p$ with $p \in Z^{+}$. Denote $\lambda(a) / \lambda(b)$ by $y$, and $a-b$ by $c$; then we have $\left(2-y-y^{-1}\right) / c^{2}=0$ or -1 .

(1) If $\left(2-y-y^{-1}\right) / c^{2}=0$, then $y=1$, that is, $\lambda(a)=\lambda(b)$;

(2) if $\left(2-y-y^{-1}\right) / c^{2}=-1$, then $y=\left(2+c^{2} \pm \sqrt{4 c^{2}+c^{4}}\right) / 2$. When $c=1, \lambda(b+1) / \lambda(b)=(3 \pm \sqrt{5}) / 2$; when $c=2$, $\lambda(b+2) / \lambda(b)=(6 \pm \sqrt{32}) / 2=3 \pm 2 \sqrt{2}$. But $\lambda(b+$ 1) $/ \lambda(b)=(3 \pm \sqrt{5}) / 2$ implies $\lambda(b+2) / \lambda(b)=(\lambda(b+$ $2) / \lambda(b+1))(\lambda(b+1) / \lambda(b))=1$, or $(7 \pm 3 \sqrt{5}) / 2$. It is a contradiction! So it is impossible that $(2-y-$ $\left.y^{-1}\right) / x^{2}=-1$.

Hence, there exists nonzero $\lambda_{0} \in \mathbb{C}$ such that $\lambda(x)=\lambda_{0}$ for every $x \in \mathbb{C}$.

We can prove the following Lemmas 8 and 9 similar as Lemmas 4 and 5 in [4].

Lemma 8 (see [4], Lemma 4). Suppose $\phi \in \Phi_{n}, A$ and $B$ are $n \times n$ orthogonal $k$-potent matrices; then $\phi(A)$ and $\phi(B)$ are orthogonal.
Lemma 9 (see [4], Lemma 5). Suppose $\phi \in \Phi_{n}$; then $\phi$ are homogeneous; that is, $\phi(\lambda X)=\lambda \phi(X)$ for every $X \in S_{n}$ and every $\lambda \in \mathbb{C}$.

Corollary 10. Suppose $\phi \in \Phi_{n}, A+B, C \in S \Gamma_{n}$, and for every $\epsilon \in \Lambda, A+B+\epsilon C \in S \Gamma_{n}, \phi(B+\epsilon C)=\phi(B)+\phi(\epsilon C)$. Then $\phi(A)+\phi(B)$ and $\phi(C)$ are orthogonal.

Proof. By the assumption and Lemma 9, we have $\phi(A)+$ $\phi(B) \in \Gamma_{n}, \phi(C) \in \Gamma_{n}, \phi(A)+\phi(B+\epsilon C)=\phi(A)+$ $\phi(B)+\epsilon \phi(C) \in \Gamma_{n}$. By Lemma 3, $\phi(A)+\phi(B)$ and $\phi(C)$ are orthogonal.

Corollary 11. Suppose $\phi \in \Phi_{n}$ and $\phi\left(D_{n}\right)=D_{n}$ for arbitrary diagonal matrix $D_{n} \in M_{n}$. Then for every $i, j \in\langle n\rangle$ with $i \neq j$, $\phi\left(E_{i j}+E_{j i}+D_{n}\right)=\lambda_{i j} E_{i j}+\lambda_{i j}^{-1} E_{j i}+D_{n}$, where $\lambda_{i j} \in \mathbb{C}$ is only decided by $i$ and $j$.

Proof. Let $A=(1 / 2)\left(E_{i j}+E_{j i}+D_{n}\right), B=(1 / 2)\left(E_{i i}+E_{j j}-D_{n}\right)$, and $C=\sum_{l \neq i, j} E_{l l}$; then $A, B$ and $C$ satisfy the assumption of Corollary 10, and $\phi(A)+\phi(B)$ and $\phi(C)$ are orthogonal; that is, $\phi\left(\left(E_{i j}+E_{j i}+D_{n}\right)\right)=\alpha_{i i} E_{i i}+\beta_{i j} E_{i j}+\gamma_{j i} E_{j i}+\delta_{j j} E_{j j}+D_{n}$ for some $\alpha_{i i}, \beta_{i j}, \gamma_{j i}$, and $\delta_{j j} \in \mathbb{C}$.

Since $\left(\eta^{-1}+\eta\right)^{-1}\left[\left(E_{i j}+E_{j i}+D_{n}\right)-\left(D_{n}-\eta^{-1} E_{i i}-\eta E_{j j}\right)\right]=$ $\left(\eta^{-1}+\eta\right)^{-1}\left(\eta^{-1} E_{i i}+E_{i j}+E_{j i}+\eta E_{j j}\right) \in S \Gamma_{n}$ for arbitrary nonzero $\eta \in \mathbb{C}$ with $1+\eta^{2} \neq 0$, after applying $\phi$, we have $\left(\eta^{-1}+\eta\right)^{-1}\left[\alpha_{i i} E_{i i}+\beta_{i j} E_{i j}+\gamma_{j i} E_{j i}+\delta_{j j} E_{j j}+\eta^{-1} E_{i i}+\eta E_{j j}\right]=$ $\left(\eta^{-1}+\eta\right)^{-1}\left[\alpha_{i i} E_{i i}+\left(\beta_{i j}-1\right) E_{i j}+\left(\gamma_{j i}-1\right) E_{j i}+\delta_{j j} E_{j j}\right]+\left(\eta^{-1}+\right.$ $\eta)^{-1}\left(\eta^{-1} E_{i i}+E_{i j}+E_{j i}+\eta E_{j j}\right) \in \Gamma_{n}$. By Lemma $5, \alpha_{i i}=\delta_{j j}=0$, $\beta_{i j} \gamma_{j i}=1$.

Let $D_{n}=\sum_{l=1}^{n} x_{l} E_{l l}$, where $x_{l} \in \mathbb{C}$ for every $l \in\langle n\rangle$; then $\beta_{i j}$ is the function of $i, j$, and $x_{l}$ and denote by $\beta_{i j}\left(D_{n}\right)$ the value of $\beta_{i j}$ on $x_{1}, \ldots, x_{n}, i$, and $j$.

Fix $i, j$, and $D_{n}$ and add a free variable $x$ to $x_{l}$ for some $l \in\langle n\rangle$; then $\beta_{i j}\left(D_{n}+x E_{l l}\right)$ becomes into a map of $x$. Since $(1 /(a-b))\left(E_{i j}+E_{j i}+D_{n}+a E_{j j}\right)-(1 /(a-b))\left(E_{i j}+E_{j i}+\right.$ $\left.D_{n}+b E_{j j}\right) \in S \Gamma_{n}$ for arbitrary $a$ and $b \in \mathbb{C}$ with $a-b \neq 0$, then by $\phi\left(E_{i j}+E_{j i}+D_{n}+a E_{j j}\right)=\beta_{i j}\left(D_{n}+a E_{j j}\right) E_{i j}+\beta_{i j}^{-1}\left(D_{n}+\right.$ $\left.a E_{j j}\right) E_{j i}+D_{n}+a E_{j j}$ and $\phi\left(E_{i j}+E_{j i}+D_{n}+b E_{j j}\right)=\beta_{i j}\left(D_{n}+\right.$ $\left.b E_{j j}\right) E_{i j}+\beta_{i j}^{-1}\left(D_{n}+b E_{j j}\right) E_{j i}+D_{n}+b E_{j j}$, we can derive that $\left(\left(\beta_{i j}\left(D_{n}+a E_{j j}\right)-\beta_{i j}\left(D_{n}+b E_{j j}\right)\right) /(a-b)\right) E_{i j}+\left(\left(\beta_{i j}^{-1}\left(D_{n}+\right.\right.\right.$ $\left.\left.\left.a E_{j j}\right)-\beta_{i j}^{-1}\left(D_{n}+b E_{j j}\right)\right) /(a-b)\right) E_{j i}+E_{j j} \in \Gamma_{n}$. By Lemma 7, $\beta_{i j}\left(D_{n}+a E_{j j}\right)=\beta_{i j}\left(D_{n}+b E_{j j}\right)$ for fixed $i, j$, and $D_{n}$; that is, $\beta_{i j}\left(D_{n}+x E_{j j}\right)=\beta_{i j}\left(D_{n}\right)$ for arbitrary $x \in \mathbb{C}$. Similarly, we can prove $\beta_{i j}\left(D_{n}+x E_{i i}\right)=\beta_{i j}\left(D_{n}\right)$ for arbitrary $x \in \mathbb{C}$.

In fact, we have proved that $\beta_{i j}\left(D_{n}+x E_{i i}\right)=\beta_{i j}\left(D_{n}\right)$ and $\beta_{i j}\left(D_{n}+y E_{j j}\right)=\beta_{i j}\left(D_{n}\right)$ for arbitrary $x, y \in \mathbb{C}$ and arbitrary $D_{n}$; then $\beta_{i j}\left(D_{n}+x E_{i i}+y E_{j j}\right)=\beta_{i j}\left(D_{n}+x E_{i i}\right)\left(=\beta_{i j}\left(D_{n}+\right.\right.$ $\left.\left.y E_{j j}\right)\right)=\beta_{i j}\left(D_{n}\right)$ follows.

Since $\beta_{i j}\left(D_{n}+x E_{j j}+y E_{l l}\right)=\beta_{i j}\left(D_{n}+y E_{l l}\right)$ for fixed $i, j$, and $l$ with $l \neq i, j$, and arbitrary $x, y \in \mathbb{C}$, then $(1 /(a-b))\left(E_{i j}+\right.$ $\left.E_{j i}+D_{n}+(a-b) E_{j j}+a E_{l l}\right)-(1 /(a-b))\left(E_{i j}+E_{j i}+D_{n}+b E_{l l}\right) \in S \Gamma_{n}$ implies $\left(\left(\beta_{i j}\left(D_{n}+a E_{l l}\right)-\beta_{i j}\left(D_{n}+b E_{l l}\right)\right) /(a-b)\right) E_{i j}+\left(\left(\beta_{i j}^{-1}\left(D_{n}+\right.\right.\right.$ $\left.\left.\left.a E_{l l}\right)-\beta_{i j}^{-1}\left(D_{n}+b E_{l l}\right)\right) /(a-b)\right) E_{j i}+E_{j j}+E_{l l} \in \Gamma_{n}$. By Lemma 7, we can get $\beta_{i j}\left(D_{n}+a E_{l l}\right)=\beta_{i j}\left(D_{n}+b E_{l l}\right)$ for arbitrary $a$ and 
$b \in \mathbb{C}$ with $a-b \neq 0$; that is, $\beta_{i j}\left(D_{n}+x E_{l l}\right)=\beta_{i j}\left(D_{n}\right)$ for arbitrary $x \in \mathbb{C}$.

Until now, we have proved that $\beta_{i j}\left(D_{n}\right)=\beta_{i j}\left(\sum_{l=1}^{n} x_{l} E_{l l}\right)=$ $\beta_{i j}\left(\sum_{l=1}^{n-1} x_{l} E_{l l}\right)=\cdots=\beta_{i j}\left(x_{1} E_{11}\right)=\beta_{i j}(0)$ for arbitrary $D_{n}$; that is, $\beta_{i j}$ is only decided by $i$ and $j$.

Remark 12. The proof of Corollary 11 presents the basic procedure of proof of Theorem 1 . In order to decide the image of matrix $A$, we use Corollary 10 and the images of $B$ and $C$, which usually are diagonal matrices or some matrices with images already decided.

If $\phi$ is a weak preserver from $M_{n}$ to $M_{n}$, then Corollary 11 is also true. Let $A=E_{i j}+D_{n}, B=-\left(E_{i j}+E_{j i}+D_{n}\right)+E_{i i}$, and $C=\sum_{l \neq i, j} E_{l l}$; then we can prove $\phi(A)=a_{i i} E_{i i}+a_{i j} E_{i j}+$ $a_{j i} E_{j i}+a_{j j} E_{j j}+D_{n}$ similarly as proving $\phi\left(\left(E_{i j}+E_{j i}+D_{n}\right)\right)=$ $\alpha_{i i} E_{i i}+\beta_{i j} E_{i j}+\gamma_{j i} E_{j i}+\delta_{j j} E_{j j}+D_{n}$, and $\left(a_{i i}+1\right) E_{i i}+\left(a_{i j}-\right.$ $\left.\lambda_{i j}\right) E_{i j}+\left(a_{j i}-\lambda_{i j}^{-1}\right) E_{j i}+a_{j j} E_{j j} \in \Gamma_{n}$. Since $\alpha^{-1} A+\alpha^{-1}\left(-\left(E_{i j}+\right.\right.$ $\left.\left.E_{j i}+D_{n}\right)+\alpha E_{i i}\right)=-\alpha^{-1} E_{j i}+E_{i i} \in \Gamma_{n}$ for arbitrary nonzero $\alpha$, then the following matrix is $k$-potent:

$$
\alpha^{-1}\left[\begin{array}{ll}
a_{i i} & a_{i j}-\lambda_{i j} \\
a_{j i}-\lambda_{j i}^{-1} & a_{j j}
\end{array}\right]+\alpha^{-1}\left[\begin{array}{ll}
\alpha & 0 \\
0 & 0
\end{array}\right] .
$$

Remark 6 tells us that $a_{i i}=a_{j j}=0, a_{i j}-\lambda_{i j}=0$, or $a_{j i}-\lambda_{i j}^{-1}=$ 0 ; that is, $\phi(A)=\lambda_{i j} E_{i j}+D_{n}$, or $\phi(A)=\lambda_{i j}^{-1} E_{j i}+D_{n}, \phi(A)=$ $\lambda_{i j} E_{i j}+\lambda_{i j}^{-1} E_{j i}+D_{n}$. Similarly, we can prove $\phi\left(E_{j i}+D_{n}\right)=$ $\lambda_{i j}^{-1} E_{j i}+D_{n}, \phi\left(E_{j i}+D_{n}\right)=\lambda_{i j} E_{i j}+D_{n}$, or $\phi\left(E_{j i}+D_{n}\right)=$ $\lambda_{i j} E_{i j}+\lambda_{i j}^{-1} E_{j i}+D_{n}$. Since $D_{n}$ is arbitrary, we set $D_{n}=0$ for convenience.

If $\phi\left(E_{i j}\right)=\lambda_{i j} E_{i j}+\lambda_{i j}^{-1} E_{j i}$; then $(1 / 3) E_{i j}+(1 / 3)\left(E_{i j}+\right.$ $\left.E_{j i}+2 E_{i i}+E_{j j}\right)=(1 / 3)\left(2 E_{i j}+E_{j i}+2 E_{i i}+E_{j j}\right) \in \Gamma_{n}$ implies $(1 / 3)\left(\lambda_{i j} E_{i j}+\lambda_{i j}^{-1} E_{j i}\right)+(1 / 3)\left(\lambda_{i j} E_{i j}+\lambda_{i j}^{-1} E_{j i}+2 E_{i i}+\right.$ $\left.E_{j j}\right)=(1 / 3)\left(2 \lambda_{i j} E_{i j}+2 \lambda_{i j}^{-1} E_{j i}+2 E_{i i}+E_{j j}\right) \in \Gamma_{n}$; that is, $-2 / 9 \in \Delta$, which is a contradiction. Hence, we proved that it is impossible $\phi\left(E_{i j}\right)=\lambda_{i j} E_{i j}+\lambda_{i j}^{-1} E_{j i}$ or $\phi\left(E_{j i}\right)=\lambda_{i j} E_{i j}+\lambda_{i j}^{-1} E_{j i}$.

If $\phi\left(E_{i j}\right)=\lambda_{i j} E_{i j}$ and $\phi\left(E_{j i}\right)=\lambda_{i j} E_{i j}$, then $(1 / 2)\left(E_{i i}+E_{i j}+\right.$ $\left.E_{j i}+E_{j j}\right) \in \Gamma_{n}$ implies $(1 / 2)\left(\phi\left(E_{i j}\right)+\phi\left(E_{i i}+E_{j i}+E_{j j}\right)\right) \in \Gamma_{n}$; that is, $(1 / 2)\left(E_{i i}+2 \lambda_{i j} E_{i j}+E_{j j}\right) \in \Gamma_{n}$, which is a contradiction. Hence, we proved that $\phi\left(E_{i j}\right)=\lambda_{i j} E_{i j}$ and $\phi\left(E_{j i}\right)=\lambda_{i j}^{-1} E_{j i}$, or $\phi\left(E_{i j}\right)=\lambda_{i j}^{-1} E_{j i}$ and $\phi\left(E_{j i}\right)=\lambda_{i j} E_{i j}$.

\section{Proof of Theorem 1}

Suppose $\phi \in \Phi_{n}$, then we can derive Theorem 1 from Propositions 13, 14, and 16.

Proposition 13. Suppose $i, j \in\langle n\rangle$ with $i \neq j$; then $\phi\left(E_{i i}\right)=0$ if and only if $\phi\left(E_{j j}\right)=0$.

Proof. Suppose $\phi\left(E_{i i}\right)=0$ and $\phi\left(E_{j j}\right) \neq 0$ for some $i, j \in\langle n\rangle$ with $i \neq j$. At first, we prove that $\phi\left(a E_{i i}+E_{j j}\right)=\phi\left(E_{j j}\right)$ for arbitrary $a \in \mathbb{C}$. Since the equation is already true when $a=0$, then we assume $a \neq 0$ in the following proof.

Let $A=a^{-1}\left(a E_{i i}+E_{j j}\right), B=-a^{-1} E_{j j}$, and $C=E_{j j}$; then it is easy to verify $A, B$, and $C$ satisfying the assumption of Corollary 10. So $\phi\left(a^{-1}\left(a E_{i i}+E_{j j}\right)\right)+\phi\left(-a^{-1} E_{j j}\right)$ and $\phi\left(E_{j j}\right)$ are orthogonal. Moreover, we can derive $\phi\left(a E_{i i}+E_{j j}\right) \in \Gamma_{n}$ from $\left(a E_{i i}+E_{j j}\right)-a E_{i i} \in S \Gamma_{n}$ and $\phi\left(E_{i i}\right)=0$. Let $a^{-1}\left(\phi\left(a E_{i i}+\right.\right.$ $\left.\left.E_{j j}\right)-\phi\left(E_{j j}\right)\right)=D$, then $D$ and $\phi\left(E_{j j}\right)$ are orthogonal $k$-potent matrices. While $\phi\left(a E_{i i}+E_{j j}\right) \in \Gamma_{n}$ implies $a D+\phi\left(E_{j j}\right) \in \Gamma_{n}$; then $a D \in \Gamma_{n}$. There are two cases on $a$.

(1) If $a \notin \Lambda$, then $D=0$; that is, $\phi\left(a E_{i i}+E_{j j}\right)=\phi\left(E_{j j}\right)$;

(2) if $a \in \Lambda$, we can derive that $(1 / 3) \phi\left(a E_{i i}+E_{j j}\right)-$ $(1 / 3) \phi\left[(a-3) E_{i i}+E_{j j}\right] \in \Gamma_{n}$ from $(1 / 3)\left(a E_{i i}+E_{j j}\right)-$ $(1 / 3)\left[(a-3) E_{i i}+E_{j j}\right] \in S \Gamma_{n}$. Note that $a-3 \notin \Lambda$, so it is true that $\phi\left[(a-3) E_{i i}+E_{j j}\right]=\phi\left(E_{j j}\right)$; that is, $(1 / 3) \phi\left(a E_{i i}+E_{j j}\right)-(1 / 3) \phi\left(E_{j j}\right)=(a / 3) D \in \Gamma_{n}$. Finally, we can derive $D=0$ from $a / 3 \notin \Lambda$ and $D \in \Gamma_{n}$. At the same time, $\phi\left(a E_{i i}+E_{j j}\right)=\phi\left(E_{j j}\right)$.

Anyway, $\phi\left(a E_{i i}+E_{j j}\right)=\phi\left(E_{j j}\right)$ for arbitrary $a \in \mathbb{C}$.

Since $\left(b^{-1}+b\right)^{-1}\left(b^{-1} E_{i i}+E_{i j}+E_{j i}+b E_{j j}\right) \in S \Gamma_{n}$ for every nonzero $b \in \mathbb{C}$ with $1+b^{2} \neq 0$, then $\left(b^{-1}+b\right)^{-1}\left[\phi\left(E_{i j}+E_{j i}\right)+\right.$ $\left.\phi\left(b^{-1} E_{i i}+b E_{j j}\right)\right] \in \Gamma_{n}$, and $\left(b^{-1}+b\right)^{-1}\left[\phi\left(E_{i j}+E_{j i}\right)+b \phi\left(E_{j j}\right)\right] \epsilon$ $\Gamma_{n}$ by $\phi\left(b^{-1} E_{i i}+b E_{j j}\right)=b \phi\left(E_{j j}\right)$. While the equation $\left(b^{-1}+\right.$ $b)^{-k}\left[\phi\left(E_{i j}+E_{j i}\right)+b \phi\left(E_{j j}\right)\right]^{k}=\left(b^{-1}+b\right)^{-1}\left[\phi\left(E_{i j}+E_{j i}\right)+\right.$ $\left.b \phi\left(E_{j j}\right)\right]$ is equivalent to $b^{k-1}\left[\phi\left(E_{i j}+E_{j i}\right)+b \phi\left(E_{j j}\right)\right]^{k}=(1+$ $\left.b^{2}\right)^{k-1}\left[\phi\left(E_{i j}+E_{j i}\right)+b \phi\left(E_{j j}\right)\right]$. Note that $\phi\left(E_{i j}+E_{j i}\right)$ is the constant term of the equation; then $\phi\left(E_{i j}+E_{j i}\right)=0$ by the infinite property of $b$, and $\left(b^{-1}+b\right)^{-1} b \phi\left(E_{j j}\right) \in \Gamma_{n}$ follows. Then we can derive $\phi\left(E_{j j}\right)=0$ which is a contradiction to the assumption.

Proposition 14. Suppose $\phi\left(E_{i i}\right)=0$ for every $i \in\langle n\rangle$; then $\phi(X)=0$ for arbitrary $X \in S_{n}$.

Proof. The proof will be completed by induction on the following equation for arbitrary $X \in S_{n}$ with $X[i, i]=x_{i}$ for every $i \in\langle n\rangle$ :

$$
\phi\left(X_{\{1, \ldots, m\}}+\sum_{i=m+1}^{n} x_{i} E_{i i}\right)=0,
$$

where $1 \leq m \leq n-1$.

When $m=1,(10)$ is equivalent to $\phi\left(\sum_{i=1}^{n} a_{i} E_{i i}\right)=0$ for arbitrary $D_{n}=\sum_{i=1}^{n} a_{i} E_{i i} \in S_{n}$.

At first, by the assumption, it is already true that $\phi\left(E_{i i}\right)=0$ for every $i \in\langle n\rangle$.

Suppose $\phi\left(\sum_{j=1}^{s} a_{i_{j}} E_{i_{j} i_{j}}\right)=0$ for every $s \in\langle n-1\rangle$ with $1 \leq$ $i_{1}<\cdots<i_{s} \leq n$; then by the homogeneity of $\phi$, we just need to prove the following equation for $i_{s+1}$ with $i_{s}<i_{s+1} \leq n$ :

$$
\phi\left(\sum_{j=1}^{s} a_{i_{j}} E_{i_{j} i_{j}}+E_{i_{s+1} i_{s+1}}\right)=0 .
$$

There are two cases on $B_{s}=\sum_{j=1}^{s} a_{i_{j}} E_{i_{j} i_{j}}$.

(1) If $B_{s} \notin S \Gamma_{n}$, then there exists $l \in\langle s\rangle$ such that $a_{i_{l}} \notin \Delta$, and the following statements are true:

$$
\begin{gathered}
\left(B_{s}+E_{i_{s+1} i_{s+1}}\right)-B_{s}=E_{i_{s+1} i_{s+1}} \in S \Gamma_{n}, \\
a_{i_{l}}^{-1}\left(B_{s}+E_{i_{s+1} i_{s+1}}\right)-a_{i_{l}}^{-1}\left(B_{s}+E_{i_{s+1} i_{s+1}}-a_{i_{l}} E_{i_{l} i_{l}}\right)=E_{i_{l} i_{l}} \in S \Gamma_{n} .
\end{gathered}
$$


Note that $\phi\left(B_{s}\right)=0$ and $\phi\left(B_{s}+E_{i_{s+1} i_{s+1}}-a_{i_{l}} E_{i_{l} i_{l}}\right)=0$ by the assumption; then the following statements are true:

$$
\begin{gathered}
\phi\left(B_{s}+E_{i_{s+1} i_{s+1}}\right) \in \Gamma_{n}, \\
a_{i_{l}}^{-1} \phi\left(B_{s}+E_{i_{s+1} i_{s+1}}\right) \in \Gamma_{n} .
\end{gathered}
$$

Since $a_{i_{l}} \notin \Delta$, then $a_{i_{l}}^{-1} \notin \Delta$, and $\phi\left(B_{s}+E_{i_{s+1} i_{s+1}}\right)=0$ follows.

(2) If $B_{s} \in S \Gamma_{n}$, then we have the following statements:

$$
\begin{gathered}
B_{s}+E_{i_{s+1} i_{s+1}} \in S \Gamma_{n}, \\
\frac{1}{3}\left(B_{s}+E_{i_{s+1} i_{s+1}}\right)-\frac{1}{3}\left(-3 E_{i_{1} i_{1}}+B_{s}+E_{i_{s+1} i_{s+1}}\right)=E_{i_{1} i_{1}} \in S \Gamma_{n} .
\end{gathered}
$$

Since $a_{i_{1}}-3 \notin \Delta$; then $\phi\left(-3 E_{i_{1} i_{1}}+B_{s}+E_{i_{s+1} i_{s+1}}\right)=0$ by case 1 , and $(1 / 3) \phi\left(B_{s}+E_{i_{s+1} i_{s+1}}\right) \in \Gamma_{n}$ follows. While $\phi\left(B_{s}+E_{i_{s+1} i_{s+1}}\right) \in$ $\Gamma_{n}$, hence we get $\phi\left(B_{s}+E_{i_{s+1} i_{s+1}}\right)=0$.

Anyway, we prove $\phi\left(\sum_{j=1}^{s} a_{i_{j}} E_{i_{j} i_{j}}+E_{i_{s+1} i_{s+1}}\right)=0$; then by the induction, (10) is true for $m=1$.

Suppose (10) is true for $m \in\langle n-1\rangle$, then we prove the case on $m+1$.

Let $X_{m}=X_{[1, \ldots, m ; 1, \ldots, m]}, g=X_{[1, \ldots, m ; m+1]}, A_{n-m}=$ $\sum_{i=1}^{n-m} x_{i+m} E_{i i} \in M_{n-m}$; then we have $g^{t}=X_{[m+1 ; 1, \ldots, m]}$ and the following equation:

$$
\phi\left(\left[\begin{array}{cc}
X_{m} & 0 \\
0 & A_{n-m}
\end{array}\right]\right)=0 .
$$

We will prove the following equation which is equivalent to (10) on $m+1$ :

$$
\phi\left(\left[\begin{array}{ccc}
X_{m} & g & 0 \\
g^{t} & x_{m+1} & 0 \\
0 & 0 & A_{n-m-1}
\end{array}\right]\right)=0
$$

For arbitrary nonzero $\alpha \in \mathbb{C}$ with $g^{t} g+\alpha^{2} \neq 0$, the following $n \times n$ matrix $B$ is idempotent:

$$
B=\tau\left[\begin{array}{ccc}
\alpha^{-1} g g^{t} & g & 0 \\
g^{t} & \alpha & 0 \\
0 & 0 & 0
\end{array}\right],
$$

where $\tau=\left(\alpha^{-1} g^{t} g+\alpha\right)^{-1}$.

Note that $X_{m+1}=\left[\begin{array}{cc}X_{m} & g \\ g^{t} & x_{m+1}\end{array}\right]$ and $A_{n-m-1}$ satisfy the following equation:

$$
\begin{gathered}
\tau\left[\begin{array}{ccc}
X_{m} & g & 0 \\
g^{t} & x_{m+1} & 0 \\
0 & 0 & A_{n-m-1}
\end{array}\right] \\
-\tau\left[\begin{array}{ccc}
X_{m}-\alpha^{-1} g g^{t} & 0 & 0 \\
0 & x_{m+1}-\alpha & 0 \\
0 & 0 & A_{n-m-1}
\end{array}\right]=B .
\end{gathered}
$$

After applying $\phi$ on the above matrices, we have $\tau \phi\left(X_{m+1} \oplus A_{n-m-1}\right) \in \Gamma_{n}$ by the inductive assumption. Then $\phi\left(X_{m+1} \oplus A_{n-m-1}\right)=0$ because of the assumption of $\alpha$; that is, (10) holds for $m+1$.

Finally, we prove that $\phi(X)=0$ for every $X \in S_{n}$ by the induction.

Remark 15. If $\phi$ is a weak $k$-potence preserver from $M_{n}$ to $M_{n}$; then Propositions 13 and 14 (replacing $g^{t}$ with $h^{t}$ for arbitrary $X \in M_{n}$ in the proof of Proposition 14) hold since Corollary 10 is true under this assumption.

Proposition 16. Suppose $\phi\left(E_{i i}\right) \neq 0$ for every $i \in\langle n\rangle$, then there exist $P \in G L_{n}$ and $c \in \Lambda$ such that $\phi(X)=c P^{-1} X P$ for every $X \in S_{n}$.

Proof. The proof will be completed in the following 4 steps.

Step 1. $\phi\left(E_{i i}\right)=c_{i} E_{i i}$, where $c_{i} \in \Lambda$ for every $i \in\langle n\rangle$.

Since $\phi\left(E_{i i}\right)$ is nonzero $k$-potent, then we can derive from Lemma 4 that there exists $P_{1} \in G L_{n}$ such that $P_{1}^{-1} \phi\left(E_{i i}\right) P_{1}=$ $c_{i} E_{i i}$ for every $i \in\langle n\rangle$, where $c_{i} \in \Lambda$. It is obvious that the following map $\varphi \in \Phi_{n}$ and $\varphi\left(E_{i i}\right)=c_{i} E_{i i}$ for every $i \in\langle n\rangle$.

$$
\varphi(X)=P_{1}^{-1} \phi(X) P_{1} \text {. }
$$

Without loss of generality, we can assume $\phi\left(E_{i i}\right)=c_{i} E_{i i}$.

Step 2. $\phi\left(\sum_{i=1}^{n} a_{i} E_{i i}\right)=\sum_{i=1}^{n} a_{i} \phi\left(E_{i i}\right)$, for arbitrary diagonal matrix $\sum_{i=1}^{n} a_{i} E_{i i}$ [5].

The proof of this step can be seen in Step 3, Section 3 in

Step 3. $c_{i}=c \in \Lambda$ for every $i \in\langle n\rangle$.

Let $A=(1 / 2)\left(E_{i j}+E_{j i}\right), B=(1 / 2)\left(E_{i i}+E_{j j}\right)$, and $C=$ $\sum_{l \in<n>\backslash\{i, j\}} E_{l l}$, we can derive the following equation from Step 2 and Corollary 10:

$$
\phi\left(E_{i j}+E_{j i}\right)=\alpha_{0} E_{i i}+\beta_{0} E_{i j}+\gamma_{0} E_{j i}+\delta_{0} E_{j j},
$$

where $\alpha_{0}, \beta_{0}, \gamma_{0}, \delta_{0} \in \mathbb{C}, i, j \in\langle n\rangle$ with $i \neq j$.

Note that $p E_{i i}+q\left(E_{i j}+E_{j i}\right)+(1-p) E_{j j} \in S \Gamma_{n}$ for $p, q \in \mathbb{C}$ with $q^{2}=p(1-p)$. In fact, 0 and 1 are all the eigenvalues of this matrix. Applying $\phi$ on the matrix $q\left(E_{i j}+E_{j i}\right)+\left[p E_{i i}+\right.$ $\left.(1-p) E_{j j}\right]$, we have $H(p)=q\left(\alpha_{0} E_{i i}+\beta_{0} E_{i j}+\gamma_{0} E_{j i}+\delta_{0} E_{j j}\right)+$ $p c_{i} E_{i i}+(1-p) c_{j} E_{j j}=\left(p c_{i}+q \alpha_{0}\right) E_{i i}+q \beta_{0} E_{i j}+q \gamma_{0} E_{j i}+((1-$ p) $\left.c_{j}+q \delta_{0}\right) E_{j j} \in \Gamma_{n}$.

Since $k$ is fixed, then $\Delta$ is the finite set which contains all of eigenvalues of $H(p)$, and there exists $w \in\{c+d \mid c, d \in \Delta\}$ such that the trace of $H(p)$ is $w$ for infinite choices of $p$; that is, there exist $\left(p_{1}, p_{2}\right)$ with $p_{1} \neq p_{2}$ such that the traces of $H\left(p_{1}\right)$ and $H\left(p_{2}\right)$ are all equal to $w$; then we have the following equation:

$$
\begin{aligned}
\left(p_{1} c_{i}+q_{1} \alpha_{0}\right) & +\left(\left(1-p_{1}\right) c_{j}+q_{1} \delta_{0}\right) \\
& =\left(p_{2} c_{i}+q_{2} \alpha_{0}\right)+\left(\left(1-p_{2}\right) c_{j}+q_{2} \delta_{0}\right)
\end{aligned}
$$

which is equivalent to 


$$
\left(q_{1}-q_{2}\right)\left(\alpha_{0}+\delta_{0}\right)=\left(p_{2}-p_{1}\right)\left(c_{i}-c_{j}\right),
$$

where $q_{s}^{2}=p_{s}\left(1-p_{s}\right)$, for $s=1,2$.

Naturally, there are infinite choices of $p_{2}$ for fixed $p_{1}$ such that the above equation is true. If $\left(q_{1}-q_{2}\right) /\left(p_{2}-p_{1}\right)$ is equal to some $a \in \mathbb{C}$, where $p_{2} \neq p_{1}, p_{1}$ and $q_{1}$ are fixed, then we can derive from the following equation:

$$
\left(a^{2}+1\right) p_{2}^{2}-\left(2 a q_{1}+2 a^{2} p_{1}+1\right) p_{2}+\left(q_{1}+a p_{1}\right)^{2}=0
$$

that there are infinite choices of $p_{2}$ for constant $\left(q_{1}-q_{2}\right) /\left(p_{2}-\right.$ $\left.p_{1}\right)$ if and only if $a^{2}+1=2 a q_{1}+2 a^{2} p_{1}+1=\left(q_{1}+a p_{1}\right)^{2}=0$. While $a^{2}+1=\left(q_{1}+a p_{1}\right)^{2}=0$ and $q_{1}^{2}=p_{1}\left(1-p_{1}\right)$ imply $p_{1}=q_{1}=0$, which is a contradiction to $2 a q_{1}+2 a^{2} p_{1}+1=0$, hence $\left(q_{1}-q_{2}\right) /\left(p_{2}-p_{1}\right)$ varies with $p_{2}$.

Since $\alpha_{0}+\delta_{0}$ and $c_{i}-c_{j}$ are all fixed numbers for fixed $\phi$, then $\alpha_{0}+\delta_{0} \neq 0$ implies that there are at least two different values of $c_{i}-c_{j}=\left(q_{1}-q_{2}\right) /\left(p_{2}-p_{1}\right)\left(\alpha_{0}+\delta_{0}\right)$ for fixed $p_{1}$ and infinite choices of $p_{2}$; it is a contradiction. So $\alpha_{0}+\delta_{0}=0$ and $c_{i}=c_{j}$ follows. Hence $c_{i}=c \in \Lambda$ for every $i \in\langle n\rangle$.

Step 4. $\phi(X)=X$ for every $X \in S_{n}$.

After the discussion in Steps 1, 2, and 3, we already have the following equation:

$$
\phi\left(\sum_{i=1}^{n} a_{i} E_{i i}\right)=c \sum_{i=1}^{n} a_{i} E_{i i},
$$

where $c \in \Lambda, a_{i} \in \mathbb{C}$ for every $i \in\langle n\rangle$. Since the map $c^{-1} \phi \in$ $\Phi_{n}$, then we can assume $\phi\left(\sum_{i=1}^{n} a_{i} E_{i i}\right)=\sum_{i=1}^{n} a_{i} E_{i i}$ without loss of generality.

The proof in this step will be completed by induction on the following equation for arbitrary $X \in S_{n}$ with $X[i, i]=x_{i}$ for every $i \in\langle n\rangle$ :

$$
\begin{gathered}
\phi\left(X_{\left\{i_{1}, \ldots, i_{m}\right\}}+\sum_{j \in<n>\backslash\left\{i_{1}, \ldots, i_{m}\right\}} x_{j} E_{j j}\right) \\
=X_{\left\{i_{1}, \ldots, i_{m}\right\}}+\sum_{j \in<n>\backslash\left\{i_{1}, \ldots, i_{m}\right\}} x_{j} E_{j j},
\end{gathered}
$$

where $1 \leq i_{1}<\cdots<i_{m} \leq n$ with $2 \leq m \leq n-1$.

When $m=2$, (25) is equivalent to $\phi\left(E_{i j}+E_{j i}+D_{n}\right)=$ $E_{i j}+E_{j i}+D_{n}$ for arbitrary diagonal matrix $D_{n} \in S_{n}$ and $i$, $j \in\langle n\rangle$ with $i<j$, since $\phi$ is homogeneous. The proof will be completed in the following (1) and (2).

(1) $\phi\left(E_{i i+1}+E_{i+1 i}+D_{n}\right)=E_{i i+1}+E_{i+1 i}+D_{n}$ for every $i \in\langle n-1\rangle$.

We already derive from Corollary 11 that $\phi\left(E_{i i+1}+E_{i+1 i}+\right.$ $\left.D_{n}\right)=\lambda_{i} E_{i i+1}+\lambda_{i}^{-1} E_{i+1 i}+D_{n}$ for every $i \in\langle n-1\rangle$, where $\lambda_{i} \in \mathbb{C}$ is only decided by $i$.

Suppose the map $\rho: S_{n} \rightarrow M_{n}$ satisfies the following equation for every $X \in S_{n}$,

$$
\begin{aligned}
\rho(X)= & \operatorname{diag}\left(1, \lambda_{1}, \lambda_{1} \lambda_{2}, \ldots, \prod_{i=1}^{n-1} \lambda_{i}\right) \phi(X) \\
& \times \operatorname{diag}\left(1, \lambda_{1}^{-1}, \lambda_{1}^{-1} \lambda_{2}^{-1}, \ldots, \prod_{i=1}^{n-1} \lambda_{i}^{-1}\right)
\end{aligned}
$$

then $\rho \in \Phi_{n}$, and for arbitrary diagonal matrix $D_{n}$ and every $i \in\langle n-1\rangle, \rho\left(D_{n}\right)=D_{n}$ and $\rho\left(E_{i i+1}+E_{i+1 i}+D_{n}\right)=E_{i i+1}+$ $E_{i+1 i}+D_{n}$.

Without loss of generality, we can assume $\phi\left(E_{i i+1}+E_{i+1 i}+\right.$ $\left.D_{n}\right)=E_{i i+1}+E_{i+1 i}+D_{n}$ for every $i \in\langle n-1\rangle$ and $\operatorname{arbitrary} D_{n}$.

(2) Suppose $\phi\left(E_{i j}+E_{j i}+D_{n}\right)=E_{i j}+E_{j i}+D_{n}$ for every $i$, $j$ with $1 \leq j-i<s<n-1$; then $\phi\left(E_{i j}+E_{j i}+D_{n}\right)=$ $E_{i j}+E_{j i}+D_{n}$ for every $i, j$ with $j-i=s$.

At first, we have to prove that $\phi\left(x_{i i+1}\left(E_{i i+1}+E_{i+1 i}\right)+\right.$ $\left.x_{i+1 i+m}\left(E_{i+1 i+m}+E_{i+m i+1}\right)+D_{n}\right)=x_{i i+1}\left(E_{i i+1}+E_{i+1 i}\right)+$ $x_{i+1 i+m}\left(E_{i+1 i+m}+E_{i+m i+1}\right)+D_{n}$ for arbitrary nonzero $x_{i i+1}$ and $x_{i+1 i+m} \in \mathbb{C}$.

By the assumption, we already have the following equations:

$$
\begin{gathered}
\phi\left(x_{i i+1}\left(E_{i i+1}+E_{i+1 i}\right)+D_{n}\right)=x_{i i+1}\left(E_{i i+1}+E_{i+1 i}\right)+D_{n} \\
\phi\left(x_{i+1 i+m}\left(E_{i+1 i+m}+E_{i+m i+1}\right)+D_{n}\right) \\
=x_{i+1 i+m}\left(E_{i+1 i+m}+E_{i+m i+1}\right)+D_{n} .
\end{gathered}
$$

Let $X_{1}=x_{i i+1}\left(E_{i i+1}+E_{i+1 i}\right)+x_{i+1 i+m}\left(E_{i+1 i+m}+E_{i+m i+1}\right)+$ $D_{n}, X_{2}=x_{i i+1}\left(E_{i i+1}+E_{i+1 i}\right)+D_{n}$, and $X_{3}=x_{i+1 i+m}\left(E_{i+1 i+m}+\right.$ $\left.E_{i+m i+1}\right)+D_{n}$. Then the following statements are true

$$
\begin{gathered}
X_{1}-\left(X_{2}-a_{i+1} E_{i+1 i+1}-a_{i+m} E_{i+m i+m}\right) \in S \Gamma_{n}, \\
X_{1}-\left(X_{2}-a_{i+1} E_{i+1 i+1}-a_{i+m} E_{i+m i+m}\right)+\epsilon \sum_{l \neq i+1, i+m}^{n} E_{l l} \in S \Gamma_{n}, \\
X_{1}-\left(X_{3}-b_{i} E_{i i}-b_{i+1} E_{i+1 i+1}\right) \in S \Gamma_{n}, \\
X_{1}-\left(X_{3}-b_{i} E_{i i}-b_{i+1} E_{i+1 i+1}\right)+\epsilon \sum_{l \neq i, i+1}^{n} E_{l l} \in S \Gamma_{n},
\end{gathered}
$$

where $x_{i+1 i+m}\left(E_{i+1 i+m}+E_{i+m i+1}\right)+a_{i+1} E_{i+1 i+1}+a_{i+m} E_{i+m i+m}$ and $x_{i i+1}\left(E_{i i+1}+E_{i+1 i}\right)+b_{i} E_{i i}+b_{i+1} E_{i+1 i+1}$ are $k$-potent.

Let $A=X_{1}, B=-\left(X_{2}-a_{i+1} E_{i+1 i+1}-a_{i+m} E_{i+m i+m}\right)$, and $C=\sum_{l \neq i+1, i+m}^{n} E_{l l}$, then $A, B$, and $C$ satisfy the assumption of Corollary 10. Hence we get $\phi(A)+\phi(B)$ and $\phi(C)$ are orthogonal; that is,

$$
\begin{aligned}
\phi\left(X_{1}\right)= & X_{2}+y_{i+1} E_{i+1 i+1}+y_{i+m} E_{i+m i+m} \\
& +y_{i+1 i+m} E_{i+1 i+m}+y_{i+m i+1} E_{i+m i+1} .
\end{aligned}
$$

Similarly, we can derive the following equation from Corollary 10:

$$
\begin{aligned}
\phi\left(X_{1}\right)= & X_{3}+z_{i} E_{i i}+z_{i+1} E_{i+1 i+1} \\
& +z_{i i+1} E_{i i+1}+z_{i+1 i} E_{i+1 i} .
\end{aligned}
$$

Comparing the above two equations, we have $z_{i}=y_{i+m}=$ $0, z_{i+1}=y_{i+1}, z_{i i+1}=z_{i+1 i}=x_{i i+1}$, and $y_{i+1 i+m}=y_{i+m i+1}=$ $x_{i+1 i+m}$, that is, $\phi\left(X_{1}\right)=X_{1}+y_{i+1} E_{i+1 i+1}$.

We will prove $z_{i+1}=y_{i+1}=0$. For arbitrary nonzero $\alpha$ with $x_{i+1 i+m}^{2}+\alpha^{2} \neq 0$, let $\tau=\left(\alpha^{-1} x_{i+1 i+m}^{2}+\alpha\right)^{-1}$, and $X_{4}=$ 
$-X_{2}+\alpha^{-1} x_{i+1 i+m}^{2} E_{i+1 i+1}+\alpha E_{i+m i+m}$; then $\tau X_{1}+\tau X_{4} \in S \Gamma_{n}$ implies $\tau \phi\left(X_{1}\right)+\tau \phi\left(X_{4}\right) \in \Gamma_{n}$; that is, the following matrix is $k$-potent since $\phi\left(X_{4}\right)=X_{4}$ by the assumption

$$
\tau\left[\begin{array}{cc}
y_{i+1} & 0 \\
0 & 0
\end{array}\right]+\tau\left[\begin{array}{cc}
\alpha^{-1} x_{i+1 i+m}^{2} & x_{i+1 i+m} \\
x_{i+1 i+m} & \alpha
\end{array}\right]
$$

by Lemma 5, $y_{i+1}=0$. Hence we prove $\phi\left(X_{1}\right)=X_{1}$.

Now we prove $\phi\left(E_{i i+m}+E_{i+m i}+D_{n}\right)=E_{i i+m}+E_{i+m i}+D_{n}$.

By Corollary 11, we already have $\phi\left(E_{i i+m}+E_{i+m i}+D_{n}\right)=$ $\lambda_{i i+m} E_{i i+m}+\lambda_{i i+m}^{-1} E_{i+m i}+D_{n}$.

For arbitrary nonzero $\alpha$ with $2+\alpha^{2} \neq 0,\left(2 \alpha^{-1}+\right.$ $\alpha)^{-1}\left(E_{i i+m}+E_{i+m i}+D_{n}\right)-\left(2 \alpha^{-1}+\alpha\right)^{-1}\left(-\alpha^{-1}\left(E_{i i}+E_{i i+1}+\right.\right.$ $\left.\left.E_{i+1 i}+E_{i+1 i+1}\right)-E_{i+1 i+m}-E_{i+m i+1}-\alpha E_{i+m i+m}+D_{n}\right)=\left(2 \alpha^{-1}+\right.$ $\alpha)^{-1}\left(\alpha^{-1}\left(E_{i i}+E_{i i+1}+E_{i+1 i}+E_{i+1 i+1}\right)+\left(E_{i i+m}+E_{i+1 i+m}\right)+\left(E_{i+m i}+\right.\right.$ $\left.\left.E_{i+m i+1}\right)+\alpha E_{i+m i+m}\right)$ is idempotent.

After applying $\phi$ on the above matrices, we have $\left(2 \alpha^{-1}+\right.$ $\alpha)^{-1} \phi\left(E_{i i+m}+E_{i+m i}+D_{n}\right)-\left(2 \alpha^{-1}+\alpha\right)^{-1} \phi\left(-\alpha^{-1}\left(E_{i i}+E_{i i+1}+\right.\right.$ $\left.\left.E_{i+1 i}+E_{i+1 i+1}\right)-E_{i+1 i+m}-E_{i+m i+1}-\alpha E_{i+m i+m}+D_{n}\right)=\left(2 \alpha^{-1}+\right.$ $\alpha)^{-1}\left(\alpha^{-1}\left(E_{i i}+E_{i i+1}+E_{i+1 i}+E_{i+1 i+1}\right)+\left(E_{i i+m}+E_{i+1 i+m}\right)+\left(E_{i+m i}+\right.\right.$ $\left.\left.E_{i+m i+1}\right)+\alpha E_{i+m i+m}\right)+\left(2 \alpha^{-1}+\alpha\right)^{-1}\left(\left(\lambda_{i i+m}-1\right) E_{i i+m}+\left(\lambda_{i i+m}^{-1}-\right.\right.$ 1) $\left.E_{i+m i}\right) \in \Gamma_{n}$.

Then $\lambda_{i i+m}=1$ by Lemma 5 .

By the induction, we prove $\phi\left(E_{i j}+E_{j i}+D_{n}\right)=E_{i j}+E_{j i}+D_{n}$ for every $i, j$ with $1 \leq i<j \leq n$.

(3) Suppose (25) is true for every $s$ with $2 \leq s<m \leq n$; then we prove it holds on $m$.

For arbitrary $X \in S_{n}$ with $X[i, i]=x_{i}$ for every $i \in\langle n\rangle$, let $A, B, U, V, y_{i_{m}}$, and $\tau$ satisfy the following equations:

$$
\begin{gathered}
A=X_{\left\{i_{1}, \ldots, i_{m}\right\}}+\sum_{j \in\langle n\rangle \backslash\left\{i_{1}, \ldots, i_{m}\right\}} x_{j} E_{j j}, \\
B=X_{\left\{i_{1}, \ldots, i_{m-1}\right\}}+\sum_{j \in\langle n\rangle \backslash\left\{i_{1}, \ldots, i_{m-1}\right\}} x_{j} E_{j j}, \\
U=X_{\left\{i_{1}, \ldots, i_{m-1} ; i_{m}\right\}}, \\
V=X_{\left\{i_{m} ; i_{1}, \ldots, i_{m-1}\right\}}, \\
y_{i_{m}}=\left(X_{\left\{i_{m} ; i_{1}, \ldots, i_{m-1}\right\}} X_{\left\{i_{1}, \ldots, i_{m-1} ; i_{m}\right\}}\right)\left[i_{m}, i_{m}\right], \\
\tau=\left(\alpha^{-1} y_{i_{m}}+\alpha\right)^{-1} .
\end{gathered}
$$

Then $\tau A+\tau\left(-B+\alpha^{-1} U V+\alpha E_{i_{m} i_{m}}\right)$ is idempotent for arbitrary nonzero $\alpha$ with $y_{i_{m}}+\alpha^{2} \neq 0$. Applying $\phi$ on it, we have $\tau \phi(A)+$ $\tau \phi\left(-B+\alpha^{-1} U V+\alpha E_{i_{m} i_{m}}\right) \in \Gamma_{n}$. Let $C=-B+\alpha^{-1} U V+\alpha E_{i_{m} i_{m}}$; then by $\tau A+\tau C+\epsilon \sum_{j \in\langle n\rangle \backslash\left\{i_{1}, \ldots, i_{m}\right\}}^{E_{j j}} \in S \Gamma_{n}$ for every $\epsilon \epsilon \Lambda$, we have $\tau \phi(A)+\phi\left(\tau C+\epsilon \sum_{j \in\langle n\rangle \backslash\left\{i_{1}, \ldots, i_{m}\right\}} E_{j j}\right) \in \Gamma_{n}$.

Note that $\phi\left(\tau C+\epsilon \sum_{j \in\langle n\rangle \backslash\left\{i_{1}, \ldots, i_{m}\right\}} E_{j j}\right)=\tau C+$ $\epsilon \sum_{j \in\langle n\rangle \backslash\left\{i_{1}, \ldots, i_{m}\right\}} E_{j j}$ and $\phi(C)=C$ by the assumption; then $\tau \phi(A)+\tau \phi(C)$ and $\sum_{j \in\langle n\rangle \backslash\left\{i_{1}, \ldots, i_{m}\right\}} E_{j j}$ are orthogonal by Corollary 10; that is, $\phi(A)=Y_{\left\{i_{1}, \ldots, i_{m}\right\}}+\sum_{j \in\langle n\rangle \backslash\left\{i_{1}, \ldots, i_{m}\right\}} x_{j} E_{j j}$ for some $Y \in M_{n}$.

On the other hand, $C=-\left(X_{\left\{i_{1}, \ldots, i_{m-1}\right\}}+\right.$ $\left.\sum_{j \in\langle n\rangle \backslash\left\{i_{1}, \ldots, i_{m-1}\right\}} x_{j} E_{j j}\right)+\alpha^{-1} U V+\alpha E_{i_{m} i_{m}}=-\left(X_{\left\{i_{1}, \ldots, i_{m}\right\}}+\right.$ $\left.\sum_{j \in\langle n\rangle \backslash\left\{i_{1}, \ldots, i_{m}\right\}} x_{j} E_{j j}\right)+\alpha^{-1} U V+\alpha E_{i_{m} i_{m}}+U+V$ implies $\tau\left(Y_{\left\{i_{1}, \ldots, i_{m}\right\}}-X_{\left\{i_{1}, \ldots, i_{m}\right\}}+\alpha^{-1} U V+\alpha E_{i_{m} i_{m}}+U+V\right)=$ $\tau\left(Y_{\left\{i_{1}, \ldots, i_{m}\right\}}-X_{\left\{i_{1}, \ldots, i_{m}\right\}}\right)+\tau\left(\alpha^{-1} U V+\alpha E_{i_{m} i_{m}}+U+V\right) \in \Gamma_{n}$ by $\tau \phi(A)+\tau \phi(C) \in \Gamma_{n}$. By Lemma 5, we can derive the following equations:

$$
\begin{gathered}
Y_{\left\{i_{1}, \ldots, i_{m-1}\right\}}=X_{\left\{i_{1}, \ldots, i_{m-1}\right\}}, \\
Y\left[i_{m}, i_{m}\right]=X\left[i_{m}, i_{m}\right], \\
Y_{\left\{i_{1}, \ldots, i_{m-1}\right\},\left\{i_{m}\right\}}=\eta U, \\
Y_{\left\{i_{m}\right\},\left\{i_{1}, \ldots, i_{m-1}\right\}}=\eta^{-1} V
\end{gathered}
$$

that is, $\phi(A)=X_{\left\{i_{1}, \ldots, i_{m-1}\right\}}+\eta U+\eta^{-1} V+\sum_{j \in\langle n\rangle \backslash\left\{i_{1}, \ldots, i_{m-1}\right\}} x_{j} E_{j j}$. Let $B_{1}$ and $B_{2}$ satisfy the following equations:

$$
\begin{gathered}
B_{1}=X_{\left\{i_{2}, \ldots, i_{m}\right\}}+\sum_{j \in\langle n\rangle \backslash\left\{i_{2}, \ldots, i_{m}\right\}} x_{j} E_{j j}, \\
B_{2}=X_{\left\{i_{1}, \ldots, i_{m-2}, i_{m}\right\}}+\sum_{j \in\langle n\rangle \backslash\left\{i_{1}, \ldots, i_{m-2}, i_{m}\right\}} x_{j} E_{j j} ;
\end{gathered}
$$

then we can prove

$$
\begin{aligned}
Y_{\left\{i_{2}, \ldots, i_{m}\right\}} & =X_{\left\{i_{2}, \ldots, i_{m}\right\}}, \\
Y\left[i_{1}, i_{1}\right] & =X\left[i_{1}, i_{1}\right], \\
Y_{\left\{i_{2}, \ldots, i_{m}\right\},\left\{i_{1}\right\}} & =\beta X_{\left\{i_{2}, \ldots, i_{m}\right\},\left\{i_{1}\right\}}, \\
Y_{\left\{i_{1}\right\},\left\{i_{2}, \ldots, i_{m}\right\}}= & \beta^{-1} X_{\left\{i_{1}\right\},\left\{i_{2}, \ldots, i_{m}\right\}}, \\
Y_{\left\{i_{1}, \ldots, i_{m-2}, i_{m}\right\}} & =X_{\left\{i_{1}, \ldots, i_{m-2}, i_{m}\right\}}, \\
Y\left[i_{m-1}, i_{m-1}\right] & =X\left[i_{m-1}, i_{m-1}\right], \\
Y_{\left\{i_{1}, \ldots, i_{m-2}, i_{m}\right\},\left\{i_{m-1}\right\}} & =\gamma X_{\left\{i_{1}, \ldots, i_{m-2}, i_{m}\right\},\left\{i_{m-1}\right\}}, \\
Y_{\left\{i_{m-1}\right\},\left\{i_{1}, \ldots, i_{m-2}, i_{m}\right\}} & =\gamma^{-1} X_{\left\{i_{m-1}\right\},\left\{i_{1}, \ldots, i_{m-2}, i_{m}\right\}} .
\end{aligned}
$$

Comparing the above three sets of equations, we can get $\phi(A)=A$, which is equivalent to $(25)$ on $m$.

By the induction, we prove that $\phi(X)=X$ for arbitrary $X \in S_{n}$.

Remark 17. If $\phi$ is a weak $k$-potence preserver from $M_{n}$ to $M_{n}$, then the proof in Steps 1, 2, and 3 of Proposition 16 holds, and we prove $\phi(X)=X$ or $\phi(X)=X^{t}$ in Step 4. We omit the detailed proof since the case on $X^{t}$ is totally the same after changing relevant notations.

\section{References}

[1] C.-K. Li and S. Pierce, "Linear preserver problems," The American Mathematical Monthly, vol. 108, no. 7, pp. 591-605, 2001.

[2] M. Brešar and P. Šemrl, "Linear transformations preserving potent matrices," Proceedings of the American Mathematical Society, vol. 119, no. 1, pp. 81-86, 1993.

[3] X. Zhang, X. Tang, and C. Cao, Preserver Problems on Spaces of Matrices, Science Press, Beijing, China, 2007. 
[4] H. You and Z. Wang, " $K$-potence preserving maps without the linearity and surjectivity assumptions," Linear Algebra and Its Applications, vol. 426, no. 1, pp. 238-254, 2007.

[5] X. Song and C. Cao, "A note on $k$-potence preserving maps," Linear Algebra and Its Applications, vol. 429, no. 7, pp. 1579-1586, 2008.

[6] Z. Wang and H. You, "Maps on upper-triangular matrix algebras preserving $k$-potences," Linear Algebra and its Applications, vol. 429, no. 8-9, pp. 1915-1928, 2008.

[7] C. G. Cao and X. Zhang, "Power-preserving additive maps," Advances in Mathematics, vol. 33, no. 1, pp. 103-109, 2004. 


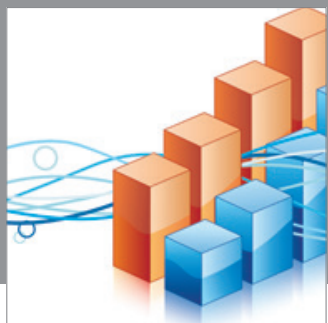

Advances in

Operations Research

mansans

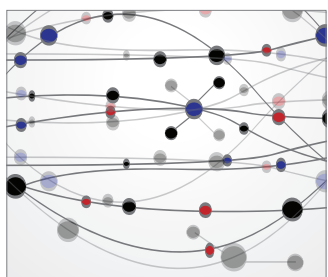

The Scientific World Journal
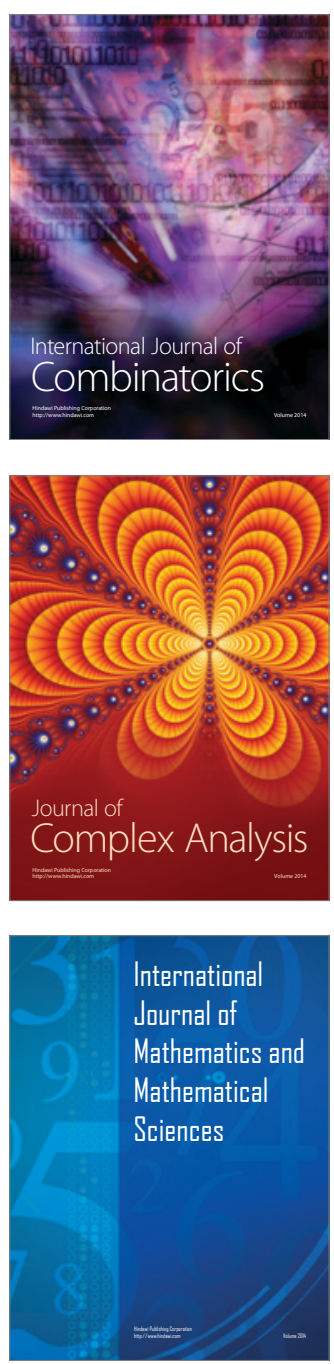
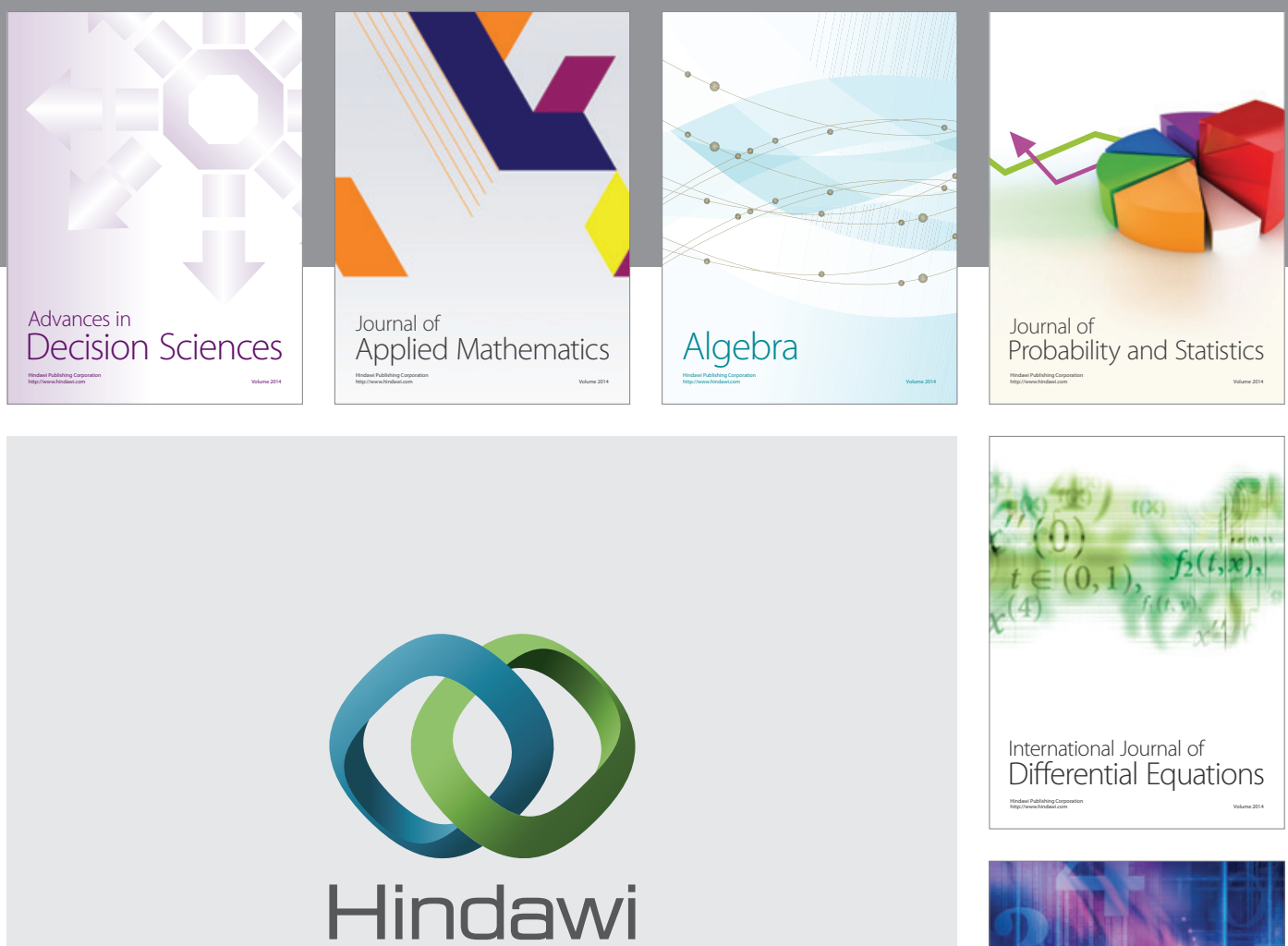

Submit your manuscripts at http://www.hindawi.com
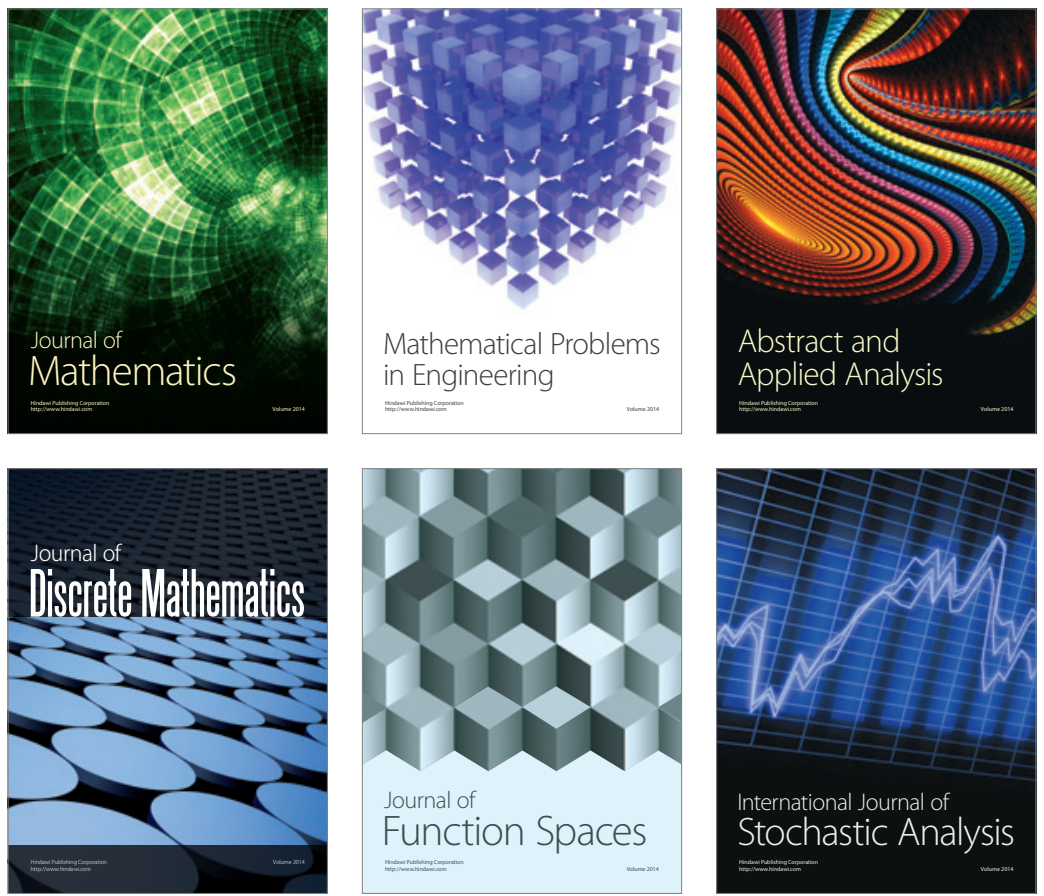

Journal of

Function Spaces

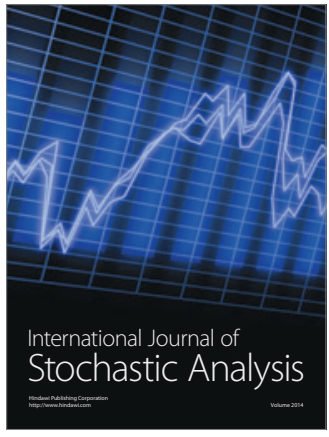

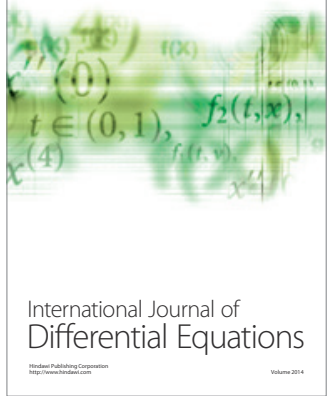
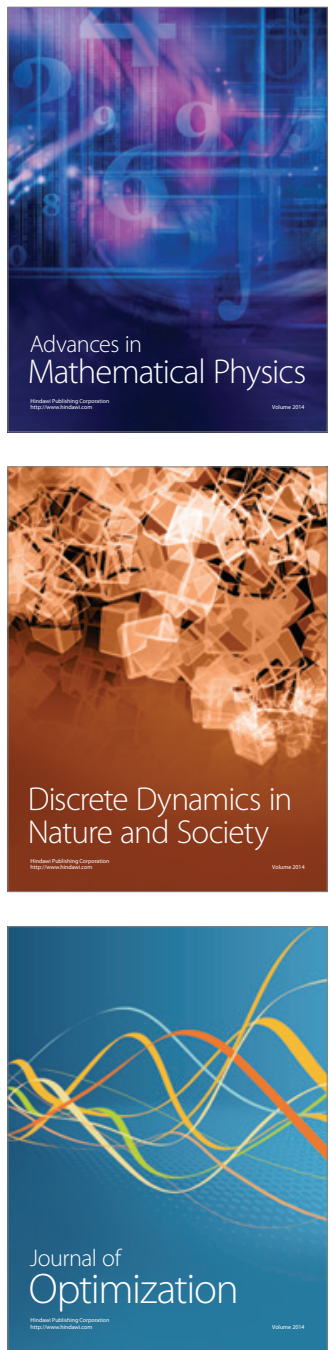